\title{
Local knowledge of traditional medicinal plants use and education system on their young of Ammatoa Kajang tribe in South Sulawesi, Indonesia
}

\author{
SUHARTINI AZIS ${ }^{1,3}$, SITI ZUBAIDAH ${ }^{1, \bullet}$, SUSRIYATI MAHANAL ${ }^{1}$, JATI BATORO ${ }^{2}$, \\ SUTIMAN B. SUMITRO ${ }^{2}$ \\ ${ }^{1}$ Department of Biology Education, Faculty of Mathematics and Natural Sciences, Universitas Negeri Malang. J1. Semarang No. 5 Malang 65145, East \\ Java, Indonesia. Tel./fax.: +62-341-551312, ”email: siti.zubaidah.fmipa@um.ac.id. \\ ${ }^{2}$ Department of Biology, Faculty of Mathematics and Natural Sciences, Universitas Brawijaya. Jl. Veteran, Malang 65145, East Java, Indonesia \\ ${ }^{3}$ Department of Biology Education, Faculty of Teacher Training and Education, Universitas Muhammadiyah Bulukumba. Jl. Poros Bulukumba-Bantaeng \\ Km. 9, Bulukumba 92561, South Sulawesi, Indonesia
}

Manuscript received: 23 June 2020. Revision accepted: 7 August 2020.

\begin{abstract}
Azis S, Zubaidah S, Mahanal S, Batoro J, Sumitro SB. 2020. Local knowledge of traditional medicinal plant use and education system on their young Ammatoa Kajang tribe in South Sulawesi, Indonesia. Biodiversitas 21: 3989-4002. Kajang tribal community in Indonesia has been using plants as traditional medicine for long periods of time; however, this traditional knowledge has not been appropriately documented. Therefore, to prevent the knowledge from being degraded or vanished, it needs to be documented. This study aimed to (i) document the local knowledge of Ammatoa Kajang tribe on the use of traditional medicinal plants, (ii) describe the education system of their young generation, and (iii) conduct Informant Consensus Factor (ICF) and Use Value (UV) analyses. The research data were obtained from 114 informants through semi-structured interviews. This research uses descriptive analysis, quantitative ICF, and UV. A total of 104 plant species from 50 families were identified. Parts of plants that were frequently used by the tribal community were leaves (48.56\%). Before using the medicinal plants, the people customarily boiled (33.09\%) the plants. The youth received education through informal education that became the highest level of education delivered orally by the family (51.75\%). The highest ICF was found in the Digestive System Diseases category, and the highest UV was observed in Piper betle.
\end{abstract}

Keywords: Education system, local knowledge, local herbalists, medicinal plant, young generation

\section{INTRODUCTION}

Traditional medicinal plants are used more in the world than in modern medicine (Prabhu and Vijayakumar 2016). Medicinal plants' usage has taken place in Asia (Wangchuk and Tobgay 2015), including in Indonesia. Traditional medicinal plant resources in Indonesia are national assets that continue to be optimally developed by exploring local knowledge. Local knowledge of traditional medicine in Indonesia is quite broad because of the high level of cultural diversity, especially in tribe communities (Sujarwo et al. 2014). The tribes in Indonesia consider that natural resources play a vital role in human life; therefore, they must be protected and used as part of the culture to ensure the balance of nature (Tamalene et al. 2016). The Ammatoa Kajang tribe is one of the local communities in Indonesia that still maintain the culture and utilizes natural resources to this day (Azis et al. 2017). The area of Ammatoa tribe is deep in the forest and grows a variety of flora that is believed and used by the community one of them as a treatment.

The Ammatoa Kajang live in groups and isolate themselves from the outside world or distance themselves from everything related to modernization (except education) (Akifah and Mukrimin 2012). Since it is difficult for them to accept changes from outside (Adhan
2005). The community believes that modernity can separate them from nature and plants are the source of life and the source of healing. The use of plants as medicine is a part of a long-standing tradition that has been empirically proven hundreds to thousands of years ago and passed on through local knowledge.

Local knowledge of the Ammatoa Kajang tribe is articulated through messages, myths, and rituals, which contain specific and essential information about plant utilization (Mithen et al. 2015). The local knowledge of medicine was passed down from their ancestors. Unfortunately, the environmental-management culture of the Ammatoa Kajang tribe only been conveyed from an oral tradition since thousands of years ago (Lullulangi et al. 2018). Therefore, era development and globalization will lead to the result in degradation, scarcity, and even the extinction of the local knowledge that only orally delivered (Srithi et al. 2009).

Local knowledge is one of the Indonesian's heritage, and the young generation is one of the parties contributing to traditional knowledge loss. The youth's tendency to be curious about medicinal plants' properties and how to cultivate them is, in fact, low (Gallois and Reyes-García 2018; Caballero-Serrano et al. 2019). The young generation also seems to ignore medicinal plants (Patrick and Tunnicliffe 2011) and lacks interest in studying traditional 
practices (Giday et al. 2009). The loss of local knowledge in the young generation occurs in some countries including America (Vandebroek and Balick 2012), North America (Saynes-Vásquez et al. 2016), Africa (Fongod et al. 2014), and Brazil (Sousa et al. 2012). The youth's poor traditional knowledge mainly results from an inadequate education system that fails to maintain local knowledge inherited from their ancestors (Khastini et al. 2019). Preserving local knowledge can be done through documentation.

Until now, local knowledge research of medicinal plants has been widely performed in the World including America (Thomas et al. 2011), Ethiopia (Kefalew et al. 2015), Pakistan (Jan et al. 2017), Myanmar (Ong et al. 2018), and India (Ju et al. 2019). In addition, some areas in Indonesia have done so between Kalimantan (Supiandi et al. 2019), Bengkulu (Wiryono et al. 2019), Banten (Khastini et al. 2019), Aceh (Suwardi et al. 2020), and Sumatera (Silalahi et al. 2019). But unfortunately local knowledge in the region of South Sulawesi especially the people of Ammatoa Kajang people have not been documented. The documentation of the medicinal plant knowledge is can encourage medical practitioners to improve the use of medicine traditionally (Yirga and Zeraburk 2011), utilized as a basic data to formulate sustainable conservation (Song et al. 2014) and provide insights into environmental management strategies and add to the archipelago's cultural wealth (Al-Fatimi 2019). This study aimed to (i) documentation the local knowledge of medicinal plant species used by the Ammatoa Kajang tribe, (ii) describe the youth education system held in the tribe, and (iii) knowing the analysis of Informant Consensus Factor (ICF) and Use Value (UV).

\section{MATERIALS AND METHODS}

\section{Study area}

Ammatoa Kajang is a sedentary tribe. They live permanently in Tanah Towa Village, Kajang Sub-district, Bulukumba District, South Sulawesi Province, Indonesia (Figure 1). Tanah Towa is about $230 \mathrm{~km}$ from Makassar, the capital city of South Sulawesi. Tanah Towa is located at $5^{\circ} 19^{\prime} 18^{\prime \prime}$ South $120^{\circ} 17^{\prime} 45^{\prime \prime}$ East. Tanah Towa has an area of about $1.698 \mathrm{ha} / \mathrm{m}^{2}$, surrounded by Batunilamung Village as the northern border, Malleleng Village as the eastern border, Bonto Baji Village as the southern border, and Pattiroang Village as the western border. Tanah Towa is divided into nine hamlets, namely Sobbu, Benteng, Pangi, Tombolo, Lurayya, Balambina, Jannaya, Balagana, and Bongkina. Tanah Towa is a low-lying area with an altitude of 200 meters above sea level. This area has an average rainfall of $13-29^{\circ} \mathrm{C}$ and humidity of $70 \%$ per year.
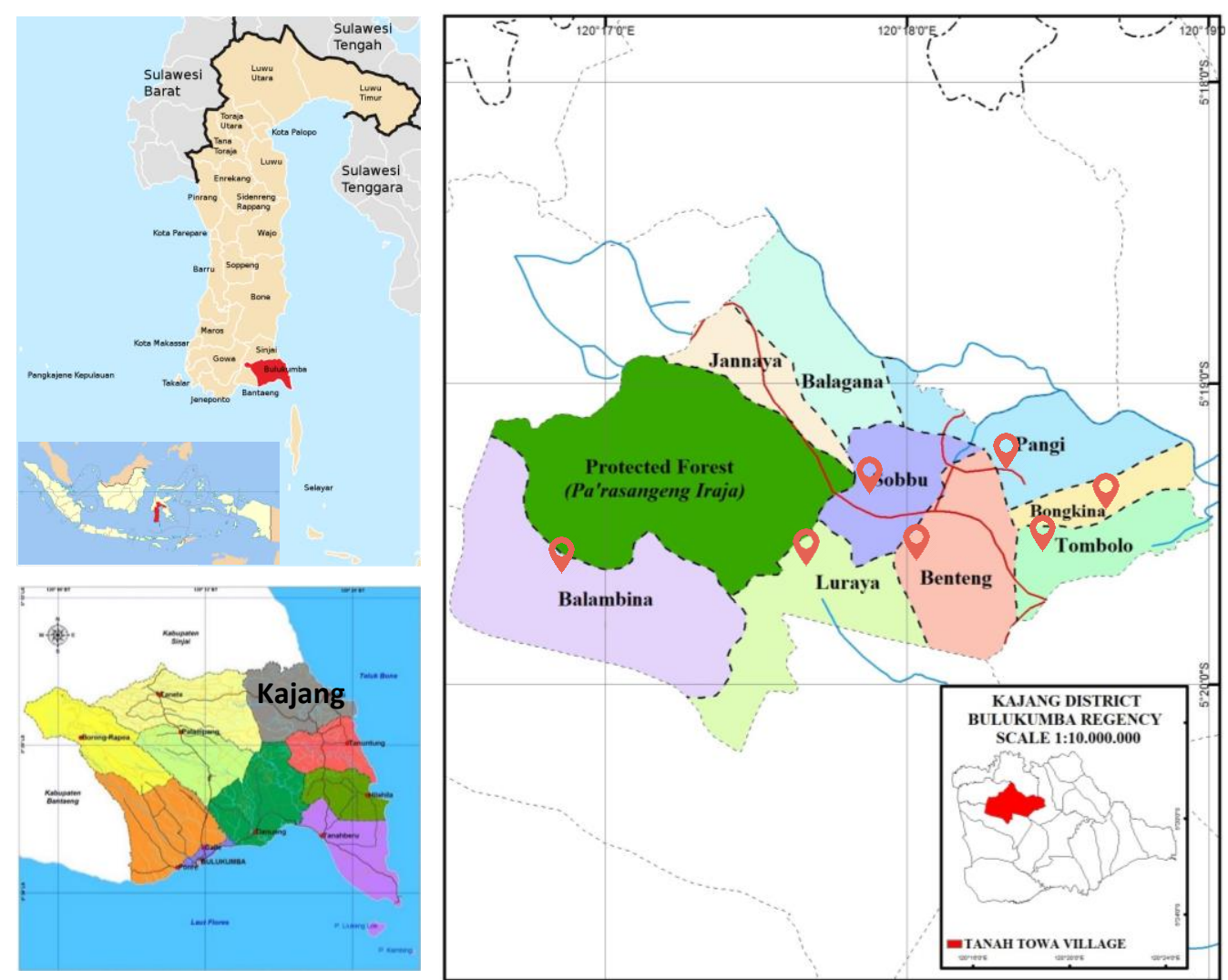

ADMINISTRATIVE MAP TANAH TOWA VILLAGE, KAJANG DISTRICT
BLLIKINGBA REGENCY BILUKUNBBA REGENCY SOUTH SILAWESI, INDONESIA 2019

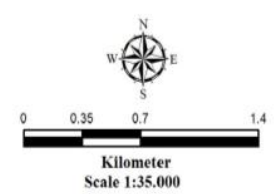

Koordinat : Geography Coordinate System Datum : Word Geodetic Svstem 1984



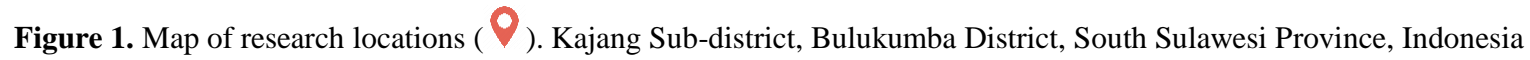


The Ammatoa Kajang community is divided into two groups, the Inner and Outer regions' groups. The Inner region group consists of Ammatoa Kajang's natives, who still live based on Pasang (rules). Their predecessors inhabited seven hamlets in Tana Towa, i.e., Sobbu, Benteng, Pangi, Buttono, Bongkina, Lurayya, and Balambina. Their custom activities are centered on Benteng, where their traditional leader (referred to as Amma) resides. On the other hand, the Outer region group consists of Ammatoa Kajang's natives who no longer practice Pasang. They are more likely influenced by modern culture. These people can be found in Balagana and Jannaya hamlets. The current study focused on the Inner region group. Areas inhabited by the Ammatoa Kajang tribe can also be referred to as Kamase-mase (simple) lands, but not all people who come from Tanah Towa belong to the Ammatoa Kajang tribal community (Nas et al. 2019). The Ammatoa Kajang tribal community can be identified by their consistent use of Pasang (rules) (Syamsurijal 2014). This study was focused on the hamlets found in the inner area community. The inner area or known as the forest is divided into three parts, namely community forests (Borong Luara), border forests (Borong Batasayya), and sacred forests (Borong Karamaka). Plants that grow in the border forest and sacred forest may only be taken by traditional stakeholders who have previously received permission from Amma (the tribal chief). The most difficult problem faced when identifying plants in the forest was due to the chief's permission.

\section{Procedures}

\section{Selection of the informants}

The informants of this study were selected from the Ammatoa Kajang tribal community using the snowball sampling technique. Snowball sampling is a technique used to collect data from a core source that can develop into several branches. The sample was determined based on information obtained from the key informants (the tribal chief, village head, customary leaders, and other trusted sources who understood the research matters, such as the Sanro (local herbalists). A total of 114 informants who represented demographic characteristics were invited to the interviews.

\section{Botanical identifications}

Data collection on the use of traditional medicinal plants by the Ammatoa Kajang tribal community was continued with the identification of the plants. The scientific name and family of each plant were identified immediately after obtaining their local names. If the scientific name could not be assigned, the plant was identified with herbarium. During the research process, the plants were photographed, and the dried specimens of the plants were collected. The collection was only made for certain plants because the retrieval of some plants was highly restricted by Pasang (rules) due to their sacredness. However, the local name, the usage, and the parts of the plants used by the community were recorded in this research. The medicinal plants were identified based on information obtained from books and herbarium institutions. Books used to determine the plants included Flora of Malesiana and Flora of Java. Furthermore, the determination of the nomenclature and the correct species quote was based on online databases, including the plant list (http://www.theplantlist.org) and plantamor (http://www.plantamor.com).

\section{Data collection}

Snowball sampling technique combined with a survey using a semi-structured interview using Konjo language (the local language). These data consisted of the community's demographic information, the local name of the plants used, parts of the plants used, methods for processing the plants, and diseases treated by the plant (Cunningham 2001). Additionally, data on the education system in young generations are obtained through semistructured interviews. This research was conducted for eight months, starting in May 2018 until January 2019.

\section{Data analysis}

Quantitative and descriptive data analyses were performed. The results of the descriptive analyses were presented in tables or pictures explaining the informants' demographic characteristics and inventory of the plants and education system on their young generation. The education system Data in their young community is analyzed descriptively (percentages) by comparing the information obtained from each respondent. Furthermore, quantitative data analysis was performed using the Informant Consensus Factor (ICF) and Use Value (UV).

\section{Informant consensus faktor (ICF)}

ICF can be calculated using the following formula: $\mathrm{ICF}=\frac{\mathrm{N}_{\mathrm{ur}}-\mathrm{N}_{\mathrm{t}}}{\mathrm{N}_{\mathrm{t}}-1}$

(Trotter and Logan 1986). ICF stands for Informant Consensus Factor, $\mathrm{N}_{\text {ur }}$ is the amount of reported usage of plants (close to 0 are categorized into the low category which suggests that there is no proper exchange of informants), and $\mathrm{N}_{t}$ is the number of taxa used by all informants. The ICF values range between $0-1$. Any values close to 1 are categorized into the high category, which means that there is a good exchange of information on medicinal plants among the people surveyed, while any values information on medicinal plants among the community members (Gazzaneo et al. 2005). Diseases treated by the medicinal plants reported in this research were sorted and grouped according to 14 categories modified from the International Classification of Primary Care (ICPC) (Miller et al. 2009), Integument Diseases (InD), Animal Bites (AnB), Circulatory System Diseases (CSD), Digestive System Diseases (DSS), Respiratory System Diseases (RSD), Reproductive System Diseases (RSS), Nervous System Diseases (NSD), Urinary System Diseases (USD), Diseases in Children (DiC), Immune System Diseases (IyS), Muscular System Diseases (MSD), Injury (Wound) Diseases (InD), Diseases in Animals (DiA), and others (Oth). The categorization of the diseases was based on the anatomical physiology system that was arranged to make it easier to examine the relationship between one ill-ness with another in the same physiological system. 


\section{Use value (UV)}

UV (use-value) analysis was performed to investigate the relative value of a plant species known locally (Tabuti 2003). UV values can be obtained using the following formula $U V=\frac{\Sigma U V i s}{i s}$ (Philips 1996), where $U V$ refers to the use-value of a plant species. UVis refers to the number of informants using the plant species and is refers to the total number of the informants. Use Value (UV) ranges between 0-100. A high UV (approaching 100) suggests that many informants use the plant species, so it can be concluded the plant species is highly significant for the community. Conversely, a low UV (approaching 0) indicates that very few informants use the plant species, so it can be concluded that the plant species is less significant for the community.

\section{RESULTS AND DISCUSSION}

\section{Local knowledge based on the informants' demographic characteristics}

Based on sociodemography, information collection is essential because it plays a significant role in the analysis and interpretation of the information (Bouyahya et al. 2017). The 114 informants involved in this study were categorized based on their age group, sex, and education level. The demographic characteristics of the informants can be seen in Table 1. Local knowledge is part of local wisdom that is still maintained until today and will be passed on to the next generation. Local knowledge of the community varies in terms of medicine use, age, and gender.

The majority of the Ammatoa Kajang community $(63 \%)$ preferred to use traditional medicine. Knowledge about medicinal plants has been maintained by the community because they consider the use of the plants to be more effective, side-effect free, easy to find and produce, and thus affordable. Only $6 \%$ of the informants reported the use of modern medicine because it is thought to be less effective and it takes time to process. Besides, about $45 \%$ of the people claimed that they used both traditional and modern medicines to support their health because they believed that the combination of both could help them recover more quickly. The results of this study differ in the North-West community in Morocco reporting a traditional treatment with a more commonly used modern treatment (Bouyahya et al. 2017).

In terms of age, it was reported that the elderly (44.74\%) from the Ammatoa Kajang tribal community possessed more knowledge about medicinal plants, followed by the adults $(30.70 \%)$, and the adolescents $(24.56 \%)$. This can happen because they have a strong belief to hold tight to the teachings passed by their ancestors. Also, adolescents who are already affected by modernity are more likely to be less aware and interested in knowing the benefits of traditional medicinal plants. These results are similar to the data on the southern slope of Mount Merapi, Yogyakarta, Indonesia (Nahdi and
Kurniawan 2019a), the Nigerian community of Awwasoma (Alade et al. 2016) where members of the community with age parents have gained a better knowledge of the use of traditional medicine compared to adolescents.

The use of medicinal plants is better known by the elderly and adult members of the community since they still have high cultural awareness and hold fast to the teachings given by their ancestors. On the other hand, teenagers are more likely to be less aware and less interested in knowing the benefits of traditional medicinal plants because they have been affected by modernity. Lack of local knowledge will have an impact on the degradation and loss of cultural identity and values. The lack of knowledge possessed by the adolescents will lead to the loss of their cultural identity and values as well as threaten the existence of traditional medicinal plants in their environment (Huai and Pei 2004).

The results of the analysis also indicated a difference in the community members' local knowledge based on gender. The tendency of using traditional medicinal plants by men is higher than that of women. Men play a significant role in family health management, so they need to be more active in collecting traditional medicines for themselves and for their families. In addition, men are more agile in discovering the presence of medicinal plants, while women are more interested in household chores, such as cooking. The findings are in line with a study in the Bajaur Agency in Pakistan Community which suggests that unlike women, men are more interested in utilizing plants as a medicine (Aziz et al. 2017), but differ in the Gunung Kidul community Yogyakarta, Indonesia, where women are more active in using medicinal plants than men (Nahdi et al. 2019b).

Table 1. The characteristics of the informants' demography

\begin{tabular}{lll}
\hline \multicolumn{1}{c}{ Categories informant } & Total & $\begin{array}{c}\text { Percentage } \\
(\%)\end{array}$ \\
\hline Medicine use & 63 & 55.26 \\
$\quad$ Traditional medicine use only & 6 & 5.26 \\
$\quad$ Modern medicine use only & 45 & 39.47 \\
Use modern and traditional medicine & 4 & \\
Age & 28 & 24.56 \\
Adolescent (13-19 Years) & 35 & 30.70 \\
Adult (20-40 Years) & 51 & 44.74 \\
Elderly (> 41 Years) & & \\
Gender & 62 & 54.39 \\
Male & 52 & 45.61 \\
Female & & \\
Degree & 26 & 24.561 \\
$\quad$ No school & 23 & 21.930 \\
$\quad$ Didn't pass Elementary & 27 & 23.684 \\
Elementary graduated & 20 & 17.544 \\
Junior high school graduated & 13 & 9.649 \\
$\quad$ Senior high school graduated & 5 & 2.632 \\
$\quad$ University graduated & & \\
\hline
\end{tabular}


Table 2. Medicinal plants used by the Ammatoa Kajang tribal community

\begin{tabular}{|c|c|c|c|c|c|c|c|c|}
\hline Scientific name & Local name & $\begin{array}{l}\text { Voucher } \\
\text { number }\end{array}$ & Status & $\mathbf{U V}$ & $\begin{array}{l}\text { Part } \\
\text { used }\end{array}$ & $\begin{array}{l}\text { Mode of the } \\
\text { preparation }\end{array}$ & $\begin{array}{l}\text { Method of } \\
\text { the uses }\end{array}$ & Ailments \\
\hline \multicolumn{9}{|l|}{ Acanthaceae } \\
\hline Andrographis paniculata (Burm. f.) Wall. ex Nees & Sombere & SA086 & Wild & 0.46 & LF & $\mathrm{Bi}$ & Drink & Malaria \\
\hline Barleria cristata $\mathrm{L}$. & Buno bатро & SA116 & Wild & 0.44 & LF & $\mathrm{Bi}$ & Drink & Gastritis \\
\hline Barleria prionitis L. & Pai-pai (balle) & SA099 & Wild & 0.68 & LF & $\mathrm{Sq}$ & Drink & Gastritis. low back pain \\
\hline Graptophyllum pictum (L.) Griffith & Lahunga & SA098 & Wild & 0.55 & LF & Po & Rub & Vulnus excoriasi \\
\hline Justicia gendarussa Burm. & Bullu-bullusu & SA083 & Cultivation & 0.61 & LF & Po & Rub & Blow air \\
\hline \multicolumn{9}{|l|}{ Amaranthaceae } \\
\hline Amaranthus spinosus $L$. & Tambara & SA131 & Wild & 0.54 & ST & $\mathrm{Bi}$ & Drink & Back Pain \\
\hline \multicolumn{9}{|l|}{ Anacardiaceae } \\
\hline Dracontomelon dao (Blanco) Merr. \& Rolfe & Rao & SA096 & Cultivation & 0.53 & ST & $\mathrm{Bi}$ & Drink & Itchy throat and dry cough \\
\hline Koordersiodendron pinnatum Merr. & Bugisi & SA088 & Cultivation & 0.65 & ST & Po & Paste & Vulnus excoriasi in animals \\
\hline Lannea coromandelica (Houtt.) Merr. & Кита-кита & SA058 & Cultivation & 0.64 & SP & Dr & Drink & Vomiting blood \\
\hline Lannea nigritana (Scott-Elliot) Keay & Kaju monro & SA063 & Cultivation & 0.68 & LF & $\mathrm{Bi}$ & Drink & Boils \\
\hline \multirow[t]{2}{*}{ Mangifera foetida Lour. } & Pao macang & SA031 & Cultivation & 0.62 & ST & Po & Paste & Diarrheal \\
\hline & & & & & LF & $\mathrm{Sq}$ & Drink & Muscle cramp \\
\hline Mangifera indica $\mathrm{L}$. & Pao & SA030 & Cultivation & 0.54 & SP & Po & Drink & $\begin{array}{l}\text { Asthma, nausea and vomitus, hematemesis, } \\
\text { vomiting blood }\end{array}$ \\
\hline \multicolumn{9}{|l|}{ Annonaceae } \\
\hline \multirow[t]{2}{*}{ Annona muricata $\mathrm{L}$. } & Sarikaja & SA042 & Cultivation & 0.80 & $\mathrm{LF}$ & $\mathrm{Sq}$ & Paste & Fever \\
\hline & & & & & & $\mathrm{Bi}$ & Drink & Fatigue \\
\hline \multicolumn{9}{|l|}{ Apiaceae } \\
\hline Pityrogramma calomelanos (L.) Link & $\begin{array}{l}\text { Tadddung- } \\
\text { taddung balaho }\end{array}$ & SA087 & Cultivation & 0.71 & LF & $\mathrm{Sq}$ & Paste & High fever; asthma \\
\hline \multicolumn{9}{|l|}{ Apocynaceae } \\
\hline \multirow[t]{2}{*}{ Alstonia scholaris (L.) R. Br } & Kaju Rital Bote & SA082 & Cultivation & 0.69 & SP & Dr & Rub & Toothache, pneumonia, diabetes \\
\hline & bote & & & & LF & $\mathrm{Bi}$ & Mouthwash & Swollen gums \\
\hline Catharanthus roseus (L.) G. Don & Song-song & SA109 & Wild & 0.58 & LF & $\mathrm{Bi}$ & Drink & Diabetes mellitus \\
\hline \multicolumn{9}{|l|}{ Arecaceae } \\
\hline \multirow[t]{3}{*}{ Areca catechu $L$. } & Rappo & SA057 & Cultivation & 0.72 & SD & - & $\begin{array}{l}\text { Chewable } \\
\text { directly }\end{array}$ & $\begin{array}{l}\text { Asthma, tuberculosis, sprue or mouth sores, } \\
\text { hepatitis, cardiovascular }\end{array}$ \\
\hline & & & & & & $\mathrm{Cu}$ & $\begin{array}{l}\text { Chewable } \\
\text { directly }\end{array}$ & Dyspnea \\
\hline & & & & & RT & Po & Drink & Impotence, premature ejaculation \\
\hline \multirow[t]{3}{*}{ Arenga Pinnata (Wurmb) Merr. } & Inru & SA049 & Cultivation & 0.75 & FL & - & Drink & Diabetes, fatigue, wor $50 \mathrm{~m}$ \\
\hline & & & & & & $\mathrm{Br}$ & Rub & Stomach pain in farm animals \\
\hline & & & & & & $\mathrm{Bi}$ & Drink & Hypotension \\
\hline Borassus flabellifer $L$. & Tala & SA055 & Cultivation & 0.67 & LF & $\mathrm{Bu}$ & Paste & Gastroenteritis \\
\hline Cocos nucifera L. & Kaluku & SA022 & Cultivation & 0.74 & FR & - & Drink & Poisoning \\
\hline
\end{tabular}




\begin{tabular}{|c|c|c|c|c|c|c|c|c|}
\hline Cocos nucifera var. eburnean. & Kaluku eja & SA023 & Cultivation & 0.63 & ST & $\mathrm{Cu}$ & $\begin{array}{l}\text { Chewable } \\
\text { directly }\end{array}$ & Cephalalgia \\
\hline $\begin{array}{l}\text { Metroxylon sagu Rottb. } \\
\text { Asphodelaceae }\end{array}$ & Kaluku lohe & SA009 & Cultivation & 0.52 & RT & $\mathrm{Br}$ & Drink & Impotence, Premature ejaculation \\
\hline Aloe vera (L.) Burm. f. & Lila buaya & SA069 & Cultivation & 0.53 & $\mathrm{LF}$ & $\mathrm{Cu}$ & Rub & $\begin{array}{l}\text { Telogen effluvium, black spots on the face, vulnus } \\
\text { combustio }\end{array}$ \\
\hline \multicolumn{9}{|l|}{ Asteraceae } \\
\hline Ageratum conyzoides (L.) L. & Ruku-ruku bembe & SA083 & Wild & 0.66 & LF & $\mathrm{Bi}$ & Drink & Asthma, Gastritis \\
\hline Artemisia vulgaris $\mathrm{L}$. & Burangga & SA134 & Wild & 0.54 & LF & $\mathrm{Sq}$ & Paste & Vulnus excoriasi \\
\hline Carthamus tinctorius $\mathrm{L}$. & Relle & SA117 & Wild & 0.79 & FL & $\mathrm{Bi}$ & Drink & Urticaria, pharyngitis, exanthema subitum \\
\hline Chromolaena odorata (L.) R.M. King \& H. Rob. & Lahuna & SA112 & Wild & 0.64 & LF & Po & Paste & Abrasions \\
\hline Elephantopus scaber L. & Dappa-dappa & SA089 & Wild & 0.53 & LF & $\mathrm{Sq}$ & Paste & Prevents breast enlargement \\
\hline Gynura segetum (Lour.) Merr. & Lahira & SA092 & Wild & 0.61 & LF & $\mathrm{Bi}$ & Drink & Fever \\
\hline Pluchea indica (L.) Less. & Biccoro & SA076 & Wild & 0.54 & LF & $\mathrm{Bi}$ & Drink & Cause to fall sweat \\
\hline \multicolumn{9}{|l|}{ Auriculariaceae } \\
\hline Auricularia auricula-judae & Pippi eja & SA071 & Wild & 0.33 & AP & Po & Drink & Diarrheal \\
\hline \multicolumn{9}{|l|}{ Bombacaceae } \\
\hline Ceiba pentandra (L.) Gaertn. & $\begin{array}{l}\text { Kahu-kahu } \\
\text { borong }\end{array}$ & SA105 & Cultivation & 0.51 & LF & $\mathrm{Sq}$ & Rub & Simplify labor, diabetes mellitus \\
\hline \multicolumn{9}{|l|}{ Boraginaceae } \\
\hline Symphytum officinale L. & Didi bulang & SA090 & Wild & 0.53 & LF & $\mathrm{Sq}$ & Paste & Allergy \\
\hline \multicolumn{9}{|l|}{ Bromeliaceae } \\
\hline $\begin{array}{l}\text { Ananas comosus (L.) Merr. } \\
\text { Caricaceae }\end{array}$ & Pandang & SA033 & Cultivation & 0.46 & FR & $\mathrm{Gr}$ & Drink & Launch menstruation \\
\hline \multirow[t]{4}{*}{ Carica papaya $\mathrm{L}$. } & Kaliki & SA014 & Cultivation & 0.79 & RT & Po & Drink & Stomach pain in farm animals \\
\hline & & & & & FR & - & $\begin{array}{l}\text { Chewable } \\
\text { directly }\end{array}$ & Expedite defecation \\
\hline & & & & & LF & $\mathrm{Cu}$ & Blew & Buzzing ears \\
\hline & & & & & & $\mathrm{Bi}$ & Drink & Malaria \\
\hline \multicolumn{9}{|l|}{ Combretaceae } \\
\hline Terminalia bellirica (Gaertn.) Roxb. & Jaha & SA129 & Wild & 0.62 & ST & Po & Drink & Diarrheal, nausea and vomitus \\
\hline Crassulaceae & & & & & & & & \\
\hline Kalanchoe pinnata (Lam.) Pers. & Dinging-dinging & SA073 & Wild & 0.83 & $\mathrm{LF}$ & $\mathrm{Sq}$ & Paste & Very high fever, bruised from falling \\
\hline \multicolumn{9}{|l|}{ Cucurbitaceae } \\
\hline Benincasa hispida (Thunb.) Cogcun. & Boyo lompo & SA040 & Cultivation & 0.64 & FR & $\mathrm{Gr}$ & Drink & Typhus \\
\hline Momordica charantia $\mathrm{L}$. & Paria & SA038 & Cultivation & 0.69 & $\mathrm{LF}$ & $\mathrm{Bi}$ & $\begin{array}{l}\text { Chewable } \\
\text { directly }\end{array}$ & Diabetes mellitus \\
\hline \multicolumn{9}{|l|}{ Dennstaedtiaceae } \\
\hline Pteridium aquilinum (L.) Kuhn & Paku balaho & SA106 & Wild & 0.55 & LF & $\mathrm{Sq}$ & Paste & Febrille convulsions/step \\
\hline \multicolumn{9}{|l|}{ Euphorbiaceae } \\
\hline \multirow[t]{3}{*}{ Aleurites moluccanus (L.) Willd. } & Sapiri & SA059 & Cultivation & 0.82 & LF & $\mathrm{Bi}$ & Drin & Fever \\
\hline & & & & & SP & Dr & Drink & Fever, sprue or mouth sores, dysentery \\
\hline & & & & & SD & Po & Paste & Cradle crap and boils \\
\hline Antidesma bunius (L.) Spreng. & Bu'nne & SA043 & Cultivation & 0.56 & LF & $\mathrm{Bi}$ & Drink & Hypertension \\
\hline
\end{tabular}




\begin{tabular}{|c|c|c|c|c|c|c|c|c|}
\hline Euphorbia pulcherrima Willd. ex Klotzs & $\begin{array}{l}\text { Tangnging- } \\
\text { tangnging } \\
\text { balanda }\end{array}$ & SA077 & Cultivation & 0.69 & SP & Dr & Rub & Yaws and vulnus excoriasi \\
\hline \multirow[t]{3}{*}{ Jatropha curcas L. } & Tangnging- & SA081 & Cultivation & 0.67 & LF & $\mathrm{Bi}$ & Drink & Hypertension \\
\hline & tangnging & & & & & $\mathrm{Br}$ & Flush & Fatigue \\
\hline & & & & & SP & Dr & Rub & Paralysis and toothache \\
\hline \multirow[t]{2}{*}{ Phyllanthus acidus (L.) Skeels } & Jaramele & SA035 & Cultivation & 0.63 & FR & - & $\begin{array}{l}\text { Chewable } \\
\text { directly }\end{array}$ & Anemia \\
\hline & & & & & ST & $\mathrm{Bi}$ & Drink & Allergy \\
\hline \multirow{2}{*}{ Sauropus androgynus (L.) Merr. } & Manya-manya & SA053 & Cultivation & 0.67 & LF & $\mathrm{Bi}$ & Drink & Increase breast milk production \\
\hline & & & & & RT & Po & Drink & Pneumonia \\
\hline \multicolumn{9}{|l|}{ Fabaceae } \\
\hline Caesalpinia sappan $\mathrm{L}$. & Seppang & SA094 & Cultivation & 0.77 & $\mathrm{ST}$ & $\mathrm{Bi}$ & Drink & Poisoning. dysentery, tuberculosis \\
\hline Cassia alata $L$. & Kiti-kiti & SA102 & Wild & 0.65 & LF & $\mathrm{Sq}$ & Rub & Pityriasis versicolor, ringworm, athlete's foot \\
\hline Crotalaria pallida Aiton & Bangkasa & SA103 & Wild & 0.64 & LF & Po & Rub & Stomach pain in farm animals \\
\hline Desmodium triquetrum DC. & Linrapa & SA127 & Wild & 0.58 & LF & $\mathrm{Bi}$ & Drink & \\
\hline \multirow[t]{2}{*}{ Indigofera tinctoria $\mathrm{L}$. } & Tarung & SA059 & Cultivation & 0.69 & RT & Po & Drink & Asthma \\
\hline & & & & & LF & $\mathrm{Br}$ & Drink & Diarrheal \\
\hline \multirow[t]{2}{*}{ Tamarindus indica $\mathrm{L}$. } & Camba & SA036 & Cultivation & 0.77 & FR & $\mathrm{Bi}$ & Drink & Dry cough, urolithiasis \\
\hline & & & & & ST & Po & Drink & Acute asthma \\
\hline \multicolumn{9}{|l|}{ Lamiaceae } \\
\hline Ocimum basilicum $\mathrm{L}$. & Talasi & SA110 & Wild & 0.60 & LF & $\mathrm{Sq}$ & Sipping & Influenza, take a cold, diarrheal, dysentery \\
\hline Orthosiphon stamineus Benth. & Kumisi cammi & SA072 & Wild & 0.51 & LF & $\mathrm{Bi}$ & Drink & Cystitis \\
\hline \multicolumn{9}{|l|}{ Lauraceae } \\
\hline Cinnamomum burmanni (Nees \& T. Nees) Blume & Kaju tanning & SA065 & Cultivation & 0.77 & ST & $\mathrm{Br}$ & Drink & Uric acid, hypertension \\
\hline Persea americana Mill. & Apoka & SA048 & Cultivation & 0.49 & $\mathrm{LF}$ & $\mathrm{Bi}$ & Drink & Hypertension \\
\hline \multicolumn{9}{|l|}{ Liliaceae } \\
\hline Allium cepa $\mathrm{L}$. & Lasuna eja & SA045 & Cultivation & 0.74 & BL & Po & Paste & Asthma \\
\hline Allium sativum $\mathrm{L}$. & Lasuna pute & SA046 & Cultivation & 0.75 & BL & Po & Rub & Tetanus \\
\hline \multicolumn{9}{|l|}{ Malvaceae } \\
\hline \multirow[t]{2}{*}{ Hibiscus tiliaceus $\mathrm{L}$. } & Baru & SA070 & Cultivation & 0.72 & LF & $\mathrm{Bi}$ & Drink & Fever \\
\hline & & & & & & $\mathrm{Sq}$ & Paste & Fever, tonsillitis \\
\hline Sida rhombifolia L. & A'llupang & SA108 & Wild & 0.48 & $\mathrm{LF}$ & $\mathrm{Sq}$ & Paste & Boils, itching stung by insects \\
\hline \multicolumn{9}{|l|}{ Menispermaceae } \\
\hline \multirow[t]{2}{*}{ Tinospora crispa (L.) Hook. f. \& Thomson } & Tambarapai & SA066 & Cultivation & 0.74 & LF & Po & Paste & $\begin{array}{l}\text { Vulnus combustio, vulnus insivum, vulnus } \\
\text { excoriasi, laceration }\end{array}$ \\
\hline & & & & & ST & $\mathrm{Bi}$ & Rub & Rheumatoid arthritis \\
\hline \multicolumn{9}{|l|}{ Melastomataceae } \\
\hline \multirow[t]{2}{*}{ Melastoma malabathricum $\mathrm{L}$. } & Biroro & SA107 & Wild & 0.56 & LF & Po & Rub & The tongue is white in children \\
\hline & & & & & & $\mathrm{Bi}$ & Drink & Diarrheal \\
\hline \multicolumn{9}{|l|}{ Moraceae } \\
\hline Artocarpus heterophyllus Lam. & Nangka & SA039 & Cultivation & 0.58 & ST & Po & Rub & Increase breast milk production \\
\hline \multirow{2}{*}{ Ficus fistulosa Reinw. ex Blume } & Lambere susu & SA067 & Cultivation & 0.77 & LF & $\mathrm{Bi}$ & Paste & Feverish \\
\hline & & & & & & $\mathrm{Sq}$ & Rub & Increase breast milk production \\
\hline
\end{tabular}




\begin{tabular}{|c|c|c|c|c|c|c|c|c|}
\hline Ficus septica Burm. f. & Tobo-tobo & SA097 & Cultivation & 0.46 & $\begin{array}{l}\text { ST } \\
\text { LF }\end{array}$ & $\begin{array}{l}\mathrm{Bi} \\
\mathrm{Sq}\end{array}$ & $\begin{array}{l}\text { Drink } \\
\text { Drink }\end{array}$ & $\begin{array}{l}\text { Libido } \\
\text { Osteoarthritis, myalgia }\end{array}$ \\
\hline \multicolumn{9}{|l|}{ Moringaceae } \\
\hline \multirow[t]{2}{*}{ Moringa oleifera Lam. } & Keloro & SA013 & Cultivation & 0.78 & LF & Po & Rub & Vernal keratoconjunctivity \\
\hline & & & & & & $\mathrm{Sq}$ & Paste & Wound from dog bite \\
\hline \multicolumn{9}{|l|}{ Musaceae } \\
\hline Musa paradisiaca $\mathrm{L}$. & Loka & SA011 & Cultivation & 0.59 & SP & Po & Rub & Vulnus excoriasi \\
\hline & & & & & LF & $\mathrm{Br}$ & Paste & Prickly heat \\
\hline \multicolumn{9}{|l|}{ Myrtaceae } \\
\hline \multirow[t]{3}{*}{ Psidium guajava $\mathrm{L}$} & Јатти bo'ddong & SA034 & Cultivation & 0.83 & LF & $\mathrm{Sq}$ & Sipping & Cold \\
\hline & (borong) & & & & & $\mathrm{Bi}$ & Drink & Diarrheal, itchy throat \\
\hline & & & & & & - & $\begin{array}{l}\text { Chewable } \\
\text { directly }\end{array}$ & Sprue or mouth sores, tonsillitis, cheilitis \\
\hline Syzygium aromaticum (L.) Merr. \& L.M. Perry & Cengke & SA044 & Cultivation & 0.57 & FR & Po & Paste & Breast cancer \\
\hline Syzygium cumini (L.) Skeels & $\begin{array}{l}\text { Rappo-rappo } \\
\text { jawa }\end{array}$ & SA052 & Cultivation & 0.70 & $\mathrm{ST}$ & $\mathrm{Bi}$ & Drink & Hypertension \\
\hline \multicolumn{9}{|l|}{ Orchidaceae } \\
\hline $\begin{array}{l}\text { Dendrobium crumenatum Sw. } \\
\text { Oxalidaceae }\end{array}$ & Tinoko & SA126 & Wild & 0.44 & ST & Po & Drops & Otitis interna, ootitis externa, tennitus \\
\hline & & & & & FR & $\mathrm{Sq}$ & Rub & Contact dermatitis \\
\hline \multicolumn{9}{|l|}{ Pandanaceae } \\
\hline $\begin{array}{l}\text { Pandanus amaryllifolius Roxb. } \\
\text { Piperaceae }\end{array}$ & Bunga & SA041 & Cultivation & 0.72 & LF & $\mathrm{Cu}$ & Flush & Fatigue \\
\hline \multirow[t]{3}{*}{ Piper betle $\mathrm{L}$. } & Leko & SA064 & Cultivation & 0.89 & $\mathrm{LF}$ & $\mathrm{Sq}$ & Paste & Fever, hives; vaginal discharge, body odor \\
\hline & & & & & & $\mathrm{Sq}$ & Drink & Dyspnea \\
\hline & & & & & & $\mathrm{Sq}$ & Sipping & Nosebleed \\
\hline \multicolumn{9}{|l|}{ Plantaginaceae } \\
\hline $\begin{array}{l}\text { Plantago major L. } \\
\text { Plumbaginaceae }\end{array}$ & Rampu-rampu & SA123 & Wild & 0.56 & LF & $\mathrm{Bi}$ & Drink & Typhus \\
\hline Plumbago zeylanica L. & Sumbila oto & SA060 & Wild & 0.44 & RT & $\mathrm{Sq}$ & Paste & Back Pain \\
\hline \multicolumn{9}{|l|}{ Poaceae } \\
\hline \multirow[t]{2}{*}{ Cymbopogon citratus (DC.) Stapf } & Serre & SA020 & Cultivation & 0.82 & RT & $\mathrm{Bi}$ & Drink & Hyperhidrosis, oliguria \\
\hline & & & & & ST & Po & Drink & Hepatitis \\
\hline Oryza sativa $\mathrm{L}$. & Pare & SA008 & Cultivation & 0.79 & SD & Po & Paste & Flatulence in children \\
\hline Oryza sativa var glutinosa "Black Sticky Rice" & Pare pulu le'lleng & SA010 & Cultivation & 0.78 & SD & $\mathrm{Bi}$ & $\begin{array}{l}\text { Chewable } \\
\text { directly }\end{array}$ & Myalgia, diabetes, anemia \\
\hline Zea mays $\mathrm{L}$. & Baddo & SA012 & Cultivation & 0.63 & FR & $\mathrm{Bu}$ & Paste & Gastroenteritis \\
\hline \multicolumn{9}{|l|}{ Portulacaceae } \\
\hline Talinum paniculatum & Munyi-munyi & SA119 & Wild & 0.46 & FR & $\mathrm{Bi}$ & Drink & Gastric acid \\
\hline
\end{tabular}


Timonius ledermannii Valeton

Uncaria gambir (Hunter) Roxb.

Rutaceae

Citrus aurantiifolia (Christm.) Swingle Citrus hystrix DC.

Murraya paniculata L.Â Jack

Sapindaceae

Nephelium lappaceum $\mathrm{L}$.

Schleichera oleosa (Lour.) Merr.

Sapotaceae

Manilkara zapota (L.) P. Royen

Scrophulariales

Justicia adhatoda $\mathrm{L}$.

Selaginellaceae

Selaginella doederleinii Hieron

Simaroubaceae

Brucea javanica (L.) Merr.

Solanaceae

Capsicum frutescens $\mathrm{L}$.

Nicotiana tabacum $\mathrm{L}$.

Sterculiaceae

Kleinhovia hospita L.

$B a e^{\prime}$

Gambere

SA056

Cultivation

Cultivation

$\begin{array}{lll}0.79 & \text { LF } & \text { Po } \\ 0.63 & \text { LF } & \text { Po }\end{array}$

Lemo kacci

Lemo lohe

SA061

SA061 Cultivation

Cultivation

Cultivation

$\begin{array}{lll}0.68 & \text { FR } & \mathrm{Br} \\ 0.39 & \mathrm{FR} & \mathrm{Wr}\end{array}$

0.24 FR Po

SA047 Cultivation

Balatung

0.42 ST Po

Kadieng

So manila

SA068

Cultivation

0.6

Galiri

Pasassa lahu

SA115

Wild

SA078

Wild

Puru

SA125 Wild

Pesse manu

SA037 Cultivation

Tambako

SA054

Cultivation

$$
0.59 \quad \mathrm{LF} \quad \mathrm{Sq}
$$

0.63 LF $\mathrm{Bi}$

$0.64 \quad$ LF $\quad \mathrm{Sq}$

0.57 FR Po

Paliasa

SA095

Cultivation

$\begin{array}{ll}\text { LF } & \text { Po } \\ \text { LF }\end{array}$

$0.56 \quad \mathrm{LF} \quad \mathrm{Bu}$

$0.78 \quad \mathrm{LF} \quad \mathrm{Bi}$

$\mathrm{Bi}$
$\mathrm{Bi}$
$\mathrm{Sq}$

Urticaceae

Poikilospermum suaveolens (Blume) Merr.

Verbenaceae

Clerodendron serratum (L.) Spr.

Lantana camara $\mathrm{L}$.

Stachytarpheta jamaicensis (L.) Vahl

Zingiberaceae

Curcuma longa $\mathrm{L}$.

Usa

Pe'ngo

Carru-carru

SA085 Wild

Bakka'ru

SA101 Wild

SA114 Wild

SA128 Wild

Didi

SA061 Cultivation

Passe pempeng SA050 Cultivation

Zingiber officinale Roscoe

Votes: LF: leaf; ST: stem; FR: fru
Drink Gastritis, gonorrhea

Drink Diarrheal

Chewable Cardiovascular, halitosis, tuberculosis, sprue or

directly

mouth sores, hepatitis

Drink Cough with phlegm

Paste Smoothing facial skin

Paste Rheumatism

Drink Diarrheal

Drink Cough

Drink Typhus

Rub Malnutrition

Drink Dizzy

Sipping Very high fever

Paste Boils

Drops Toothache

Paste Boils

Suction Cardiovascular, hepatitis

Paste Itching stung by insects

Paste Jaundice

Drink Hepatitis, cardiovascular, hypertension

Rub Contact dermatitis, athlete's foot

Rub Contact dermatitis, athlete's foot

Drink Gastritis

Paste Obesity

Paste Diarrhea in children

Paste Flatulence in children

Drink Dysmenorrhea

Paste Gastritis 

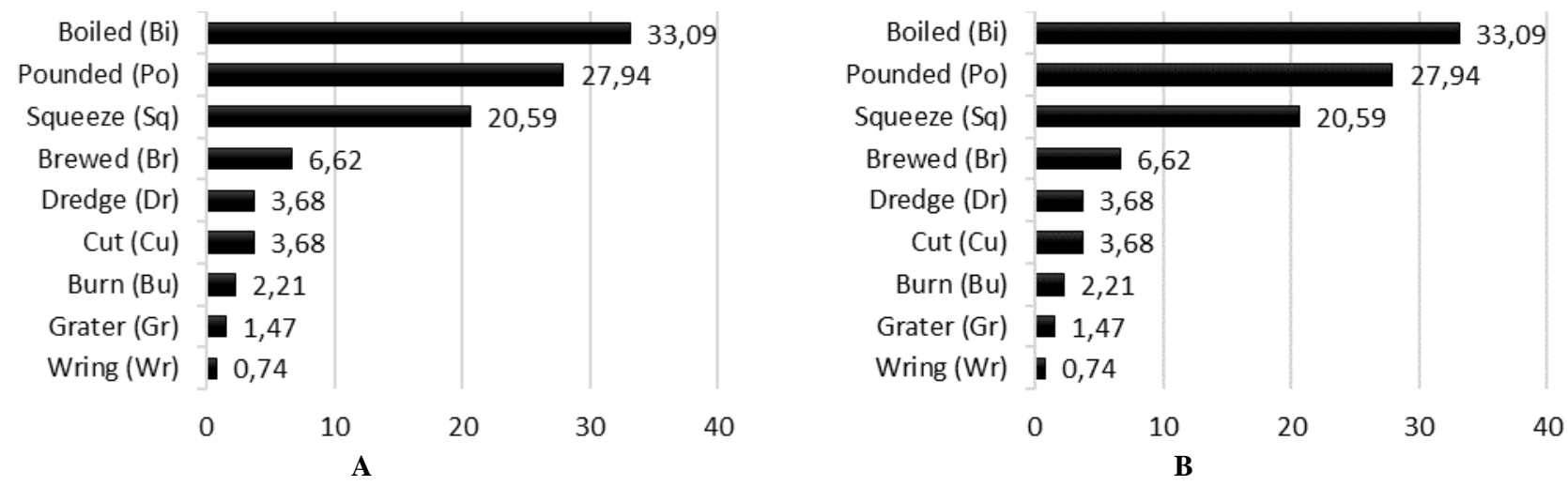

Figure 2. Percentage (A) parts used and (B) mode of preparation in traditional medicines

Traditional medicinal plants Ammatoa Kajang Community Diversity of plant species used

The Ammatoa Kajang tribal community used a total of 104 plant species from 50 families as medicinal plants in Table 2. These medicinal plants mostly come from the Asteraceae families (6.73\%), then Anacardiaceae, Arecaceae, Euphorbiaceae, and Fabaceae (each of 5.77\%).

Plant family from Asteraceae, Anacardiaceae, Fabaceae, Euphorbiaceae, and Arecaceae can be easily found in their surroundings and thus have a significant contribution to the community's health. The Asteraceae family is also mostly used by people in some areas of Indonesia including Poncokusumo in East Java Province (Batoro and Siswanto 2017), different to the Tobelo community in the province of North Maluku dominant using the family Euphorbiaceae (Yakub et al. 2019) and the village Dayak community in Kalimantan dominant utilizing family Araceae (Supiandi et al. 2019).

All species of plants used by the Ammatoa Kajang as medicinal materials obtained both through cultivation and wild (Table 2.). Cultivation Status $(64.42 \%)$ is the most crop acquisition used by communities compared to wild plants $(35.58 \%)$. The use of the highest cultivated plants is also reported in the Baduy tribe of Indonesia (Khastini et al. 2019). The most culturally cultured plants are used by the people of Ammatoa because the community preserves the plants mostly and believes that the plants are part of their lives.

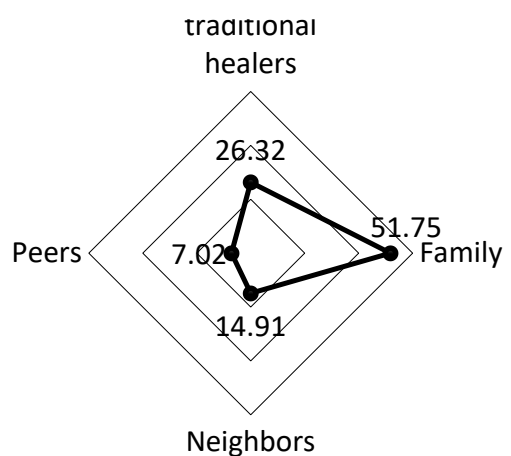

Figure 3. The percentage of informal education system pathway local knowledge Ammatoa Kajang

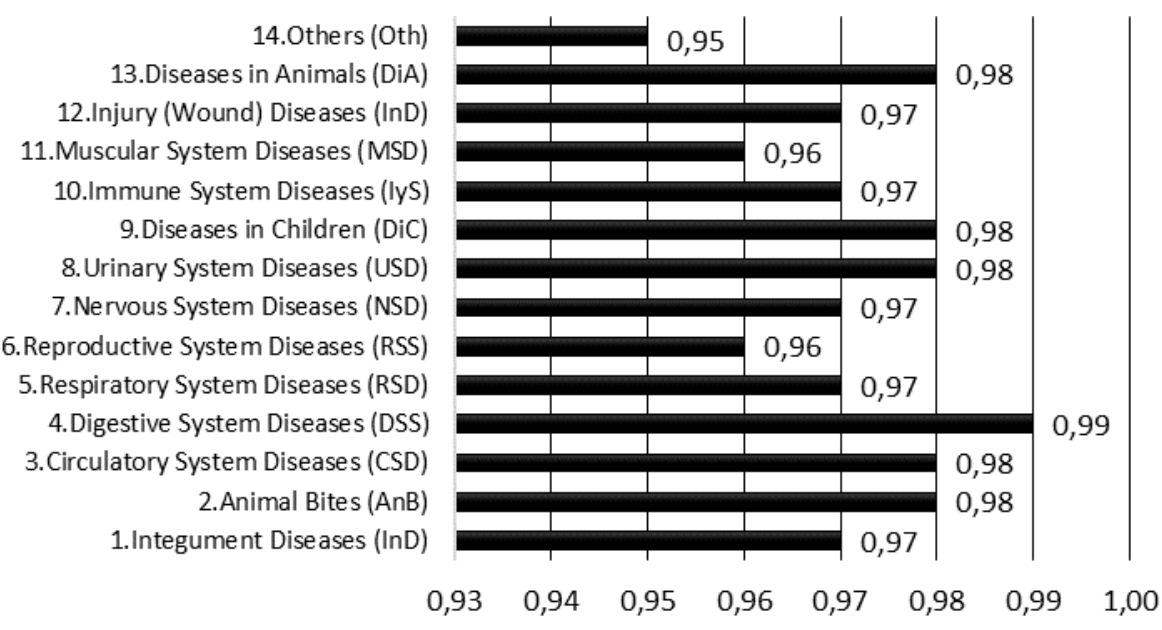

Figure 4. Category of diseases and ICF values 


\section{Plant parts used and mode of preparation in traditional medicines}

Parts of plants used by the Ammatoa Kajang community for remedies include the leaf, stem, fruit, rhizome, bulb, flower, seed, sap, and root (Figure 2).

Leaf part $(48.56 \%)$ is the highest part used and followed by a section of the stem (15.93\%), and the rhizome section $(0.88 \%)$. The lowest used by the community of Ammatoa Kajang. This result is similar to the data obtained in Indonesia for the Ranggawulung West Java, Indonesia (Putri et al. 2016), but different from the Minangkabau community in Nagari Pagaruyung, West Sumatra, which has more to tighten the plant stem as a medicine (Monita et al. 2018). Leaves are the center of bioorganic metabolism and provide storage for secondary metabolites (Bouyahya et al. 2017). Leaves also play a crucial role in photosynthesis (Ghorbani 2005), and therefore, they contain a lot of secondary bioactive compounds that aim to defend themselves from herbivores (Bhattarai et al. 2006). To avoid deforestation, the Ammatoa Kajang community only use young shoots. They keep themselves from damaging plant roots because they realize this will be able to kill the plant. The dosage given depends on age; for example, 3-5 leaves for children and 79 leaves for adults.

Traditional medicine plant preparation mode varies among boiling, pounded, squeeze, brewed, dredge, cut, burn, grater, and wring (Figure 2.). The preparation of traditional medicinal plants of the highest ethnic community was carried out through boiling mode $(33.09 \%)$, and the lowest in wring mode $(0.74 \%)$. This result is similar to the data obtained in Indonesia for the Peadundung Village, North Sumatra Indonesia (Silalahi et al. 2019). The boiling method can maintain the effectiveness of herbal medicines (Kayani et al. 2015), and accelerate biological reactions so as to produce many responses of active compounds (Chen et al. 2008). Boiling herbs can be done 2-3 times a day, depending on the age or type of disease being treated. The herbs stew can be given two times a day to children (day and night), and three times a day to adults (morning, noon, night). The community depends on plants that grow in their environment as a traditional medicine because they believe in their effectiveness.

\section{The education system of their young}

The differences in the degree (educational background) of the Ammatoa Kajang people were inversely proportional to the local knowledge level (Table 1). The degree of college-level education precisely local knowledge is increasingly lacking. Likewise, the degree of no school but local knowledge is high. This proves that the degree (educational background) of the Ammatoa Kajang tribe does not affect the level of local knowledge it possesses. This is because the education gained by the public does not provide information regarding the local knowledge of medicinal plants traditionally. These results are similar to the Semende tribe Bengkulu Province, Indonesia (Wiryono et al. 2019), Waorani community in Ecuador (Weckmüller et al. 2019) where local knowledge is not influenced by the level of education caused by social-cultural change. In addition, their education level does not influence the community members' local knowledge but because of their awareness of the benefits of using plants (Jaradat et al. 2016; Caballero-Serrano et al. 2019). Most of the local community members were prohibited by Kasipalli (prohibition) to attend formal schools. However, since 1970, the law has been removed, and now the local government has attempted to encourage the tribe to receive an education. Their education level does not influence the community members' local knowledge but because of their awareness of the benefits of using plants (Jaradat et al. 2016; Caballero-Serrano et al. 2019).

The difference in the local knowledge of medicinal plants between the older and younger community members shows the erosion of knowledge. The erosion of local knowledge occurs because knowledge is only conveyed through verbal communication (oral), has no written documentation, the parent's community has diminished knowledge, and the influence of modernity. Modernity has lowered young people's appreciation of local knowledge of traditional medicinal plants. Therefore, the educational system is crucial to raising the young generation's awareness of the importance of local knowledge.

Local knowledge contains facts that must be recognized and learned from experience and observation through education that is inherited from generation to generation. The local knowledge of the Ammatoa Kajang tribal community is obtained from the older generation and inherited to the young generation through the informal education system. The informal education system was delivered to the youth orally by their customary leaders and/or traditional healers, family, neighbors, and peers. (Figure 3$)$. The family had the highest percentage $(51.75 \%)$ of contribution to the youth education system, while peers contributed the lowest percentage to the system as much as $7 \%$.

In fact, the family education system had some limitations since the knowledge of medicinal plant use was only delivered when a family member was getting sick. There were no educational demands that all children had to understand the treatment system, and the family was unable to develop the youth's interest and motivation to preserve the local knowledge. The inability of the family to give education to the local knowledge of the younger generation is one of the factors that led to the loss of such valuable information (Sousa et al. 2012). Ideally, the family plays an important role as the first education provider to transfer local knowledge to the children and make sure that the knowledge can be transferred completely to the children (Chirico 2008). Besides family, the customary leaders and/or traditional healers provided education was limited to a particular generation so that the youth had different opportunities to access the information.

All this time, the Ammatoa Kajang tribal community has obtained knowledge through informal education so that the knowledge has slowly degraded and gone extinct in the young generation. Education based on the local knowledge 
of the Ammatoa Kajang tribal community is most required. The knowledge should be preserved and developed as the ancestral cultural heritage through formal education that is motivated and aware of protecting local knowledge. The formal education curriculum should be designed in such a way to maintain local knowledge owned by particular people and deliver it to the younger generation (Ramirez 2007). An education curriculum serves as the foundation of society's sustainable development (Dziva et al. 2011). Incorporating local knowledge into an education curriculum needs to be done to maintain the local knowledge (Boafo et al. 2016), create an awareness of the values and potential contributions of local knowledge and society's practices to sustainable development (Dei et al. 2012), support national identity (Syarif et al. 2016a). The importance of local knowledge learned, maintained, and used as a filter for the impact of globalization flows (Syarif et al. 2016b).

\section{Informant consensus factor (ICF) and use value (UV) analysis \\ Informant consensus factor analysis}

According to 114 informants, there are these diseases fall into 14 categories. The categories, types of diseases, and ICF values are shown in Figure 4 . The category with the highest degree of consensus from the informants was digestive system diseases (0.99). While the ICF value in the digestive system category resides second after the respiratory system category in the Society Delanta Northern Ethiopoia (Meragiaw et al. 2016) and the digestive system is in second place after the category of skin and hair diseases in the Jazan Region Saudi Arabian people (Tounekti et al. 2019).

This study has revealed that the Ammatoa Kajang tribal community has a high diversity of traditional medicinal plants and has a high dependency on these plants due to the belief and confidence in the efficacy and effectiveness of these plants. Traditional medicinal plants play a crucial role in Ammatoa Kajang society, as indicated by the high ICF values possessed by most plants identified in this study. This finding suggests that local knowledge of the traditional medicinal plants owned by the community has been appropriately maintained until today. Medicinal plants that have the highest ICF value will be easily acquired or used by the community so that it can cause damage to the natural plant ecosystem in the future. Therefore, more studies are needed to consider a wise or alternative step in the maintenance of such crops (Song et al. 2014).

\section{Use value (UV) analysis}

The use of medicinal plant species by 114 informants showed UV values that ranged between 0.33-0.89 (Table 2 ). The highest UV, as much as 0.89 , was found in Piper betle L., followed by Psidium guajava L. (0.83) and Kalanchoe pinnata (Lam.) Pers. (0.83) and Aleurites moluccanus (L.) Willd. (0.82). On the other hand, the lowest value was observed in Auricularia auricula-judae (0.33), followed by Citrus hystrix DC. (0.34). The Ammatoa Kajang community believed that Piper betle $L$. could be used as a remedy for fever, dyspnea, and nosebleed. According to the tribe, this plant species symbolized soothing calm (Azis et al. 2017). Piper betle L. is herb that can be easily found by the community. It is also a compulsory component of the tribe's rituals. Piper betle L. contains various beneficial properties, such as Alkaloid, amino acids, steroid, tannin, terpenes (cineole, cadinene, camphene, caryophyllene, pinene, limonene, chavicol, allyl pyrocatechol, carvacrol, safrole, eugenol, and chavibetol) (Pradhan et al. 2013). The compounds in Piper betle L. have antimicrobial properties as an inhibitor of microbial and bacterial growth. Betel leaf contains essential oils whose constituent components are phenol compounds which possess anti-bactericidal, fungicidal, or germicidal properties.

\section{ACKNOWLEDGEMENTS}

The authors would like to express their sincere gratitude to Ammatoa (the tribal chief), and customary leaders especially Puto Kaharu, Galla Puto, and the Sanro (shamans) as well as all Ammatoa Kajang tribal community members who have collaborated and participated in providing information for this research. In addition, the authors also thank the local government of Tanah Towa who continues to provide extraordinary support and advice in completing the research. We are also grateful to Lembaga Penelitian dan Pengabdian kepada Masyarakat (LP2M) of Universitas Negeri Malang for Publications' Grant funding under contract No. 4.3.482/UN32.14.1/LT/2020.

\section{REFERENCES}

Adhan. 2005. Sistem Nilai Masyarakat Ammatoa. Pustaka Refleksi, Makassar. [Indonesian]

Alade GO, Okpako E, Ajibesin KK, Omobuwajo OR. 2016. Indigenous knowledge of herbal medicines among adolescents in Amassoma, Bayelsa State, Nigeria. Glob J Health Sci 8 (1): 237-257.

Akifah A, Mukrimin. 2012. Kajang; a picture of modesty: an Indonesian local belief. Al Ulum 12 (1): 117-128.

Al-Fatimi M. 2019. Ethnobotanical survey of medicinal plants in central Abyan governorate, Yemen. J Ethnopharmacol 241: 1-24.

Azis S, Zubaidah S, Mahanal S, Batoro J, Sumitro SB. 2017. Ethnobotany of traditional ceremonies in Ammatoa Kajang Tribe, South Sulawesi, Indonesia. Proceeding $1^{\text {st }}$ International of Mathematics, Science, and Education. Universitas Negeri Malang, Malang, 30 August 2017. [Indonesian]

Aziz MA, Khan AH, Adnan M, Izatullah I. 2017. Traditional uses of medicinal plants reported by the indigenous communities and local herbal practitioners of Bajaur Agency, federally administrated tribal areas, Pakistan. J Ethnopharmacol 198: 268-281.

Batoro J, Siswanto D. 2017. Ethnomedicinal survey of plants used by local society in Poncokusumo Sub-district, Malang, East Java Province, Indonesia. Asian J Med Biol Res 3 (2): 158-167.

Bhattarai S, Chaudhary RP, Taylor RSL. 2006. Ethnomedicinal plants used by the people of Manang district, central Nepal. J Ethnobiol Ethnomed 2: 1-8.

Boafo YA, Saito O, Kato S, Kamiyama C, Takeuchi K, Nikahara M. 2016. The role of traditional ecological knowledge in ecosystem services management: the case of four rural communities in Northern Ghana. Int J Biodivers Sci Ecosyst Serv Manag 12 (1-2): 24-38.

Bouyahya A, Abrini J, Et-Touys A, Bakri Y, Dakka N. 2017. Indigenous knowledge of the use of medicinal plants in the North-West of Morocco and their biological activities. Eur J Integr Med 13: 9-25. 
Caballero-Serrano V, McLaren B, Carrasco JC, Alday JG, Fiallos L, Amigo J, Onaindia, M. 2019. Traditional ecological knowledge and medicinal plant diversity in Ecuadorian Amazon home gardens. Glob Ecol Conserv 17: 1-23.

Chen G, Yang M, Song Y, Lu Z, Zhang J, Huang H, Guan S, Wu L, Guo D. 2008. Comparative analysis on microbial and rat metabolism of ginsenoside RB1 by high-performance liquid chromatography coupled with tandem mass spectrometry. Biomed Chromatograph 22: $779-785$

Chirico F. 2008. Knowledge Accumulation in family firms: evidence from four case studies. Int Small Business J 26 (4): 433- 455.

Cunningham BA. 2001. Applied Ethnobotany People, Wild Plant Use, and Conservation. Earthscan Publications Ltd., London.

Dei SGJ, Hall BL, Rosenberg DG. 2002. Indigenous Knowledges in Global Contexts: Multiple Readings of Our World. University of Toronto Press, Toronto.

Dziva D, Mpofu V, Kusure LP. 2011. Teachers' conception of indigenous knowledge in science curriculum in the context of Mberengwa District, Zimbabwe. Afr J Educ Technol 1 (3): 88-102.

Fongod AGN, Ngoh LM, Veranso MC. 2014. Ethnobotany, indigenous knowledge and unconscious preservation of the environment: an evaluation of indigenous knowledge in South and Southwest Regions of Cameroon. Int J Biodivers Conserv 6 (1): 85-99.

Gallois S, Reyes-García V. 2018. Children and ethnobiology. J Ethnobiol 38 (2): $155-169$.

Gazzaneo LRS, Paiva de Lucena RF, Paulino de Albuquerque U. 2005. Knowledge and use of medicinal plants by local specialists in a region of Atlantic forest in the state of Pernambuco (Northeastern Brazil). $J$ Ethnobiol Ethnomed 1 (1): 9

Ghorbani A. 2005. Studies on pharmaceutical ethnobotany in the region of Turkmen Sahra, north of Iran (Part 1): General results. J Ethnopharmacol 102 (1): 58-68.

Giday M, Asfaw Z, Woldu Z. 2009. Medicinal plants of the Meinit ethnic group of Ethiopia: an ethnobotanical study. J Ethnopharmacol 124 (3): 513-521. DOI: 10.1016/j.jep.2009.05.009.

Huai H, Pei S. 2004. Plants used medicinally by folk healers of the lahu people from the autonomous county of Jinping Miao, Yao, and Dai in Southwest China. Econ Bot 58: S265-S273.

Jan HA, Walia S, Ahmad L, Jan S, Ahmad N, Ullah N. 2017. Ethnomedicinal survey of medicinal plants of Chinglai valley, Buner district, Pakistan. Europ J Integr Med 13: 64-74.

Jaradat NA, Ayesh OI, Anderson C. 2016. Ethnopharmacological survey about medicinal plants utilized by Herbalists and Traditional Practitioner Healers for treatments of diarrhea in the West Bank/ Palestine. J Ethnopharmacol 182: 57-66.

Ju SK, Mj KC, Semotiuk AJ, Krishna V. 2019. Indigenous knowledge of medicinal plants used by ethnic communities of South India. Ethnobot Res Appl 18: 1-112.

Kefalew A, Asfaw Z, Kelbessa E. 2015. Ethnobotany of medicinal plants in Ada'a District East Shewa Zone of Oromia Regional State, Ethiopia. J Ethnobiol Ethnomed 11 (1): 25

Khastini RO, Wahyuni I, Saraswati I, Alimuddin. 2019. Ethnobotanical study of medicinal plants utilized by the Baduy tribe used as a learning resource. Jurnal Pendidikan Biologi Indonesia 2: 197-206. [Indonesian]

Kayani, Ahmad M, Sultana S, Shinwari ZK, Zafar M, Yaseen G, Hussain M, Bibi T. 2015. Ethnobotany of medicinal plants among the communities of Alpine and Sub-Alpine Regions of Pakistan. J Ethnopharmacol 164: 186-202.

Lullulangi MS, Sampebua O, Sunardi, Dirawan GD. 2015. Model local wisdom to preserve the environment in south Sulawesi and West Sulawesi Indonesia. Man India 95 (4): 1041-1050.

Meragiaw M, Asfaw Z, Argaw M. 2016. The status of ethnobotanical knowledge of medicinal plants and the impacts of resettlement in Delanta, Northwestern Wello, Northern Ethiopia. Evidence-Based Complement Altern Med http://dx.doi.org/10.1155/2016/5060247.

Miller GC, Britt H, O'Halloran J. 2009. International classification of primary care. Meeting of the who collaborating centres for the family of international classifications. Family Medicine Research Centre, Australia.

Mithen, Sampebua O, Sunardi, Dirawan GD. 2015. Model local wisdom to preserve the environment in South Sulawesi and West Sulawesi Indonesia. Man India 95 (4): 1041-1050.

Monita R, Dharma F, Baihaqi A, Jannatan R. 2018. An Ethnobotanical study of medicinal plants used by Minangkabau Ethnic in Nagari
Pagaruyung, West Sumatra, Indonesia. Int $\mathrm{J}$ Bot Stud. DOI: 10.13140/RG.2.2.23511.78243.

Nahdi MS, Kurniawan AP. 2019a. The diversity and ethnobotanical study of medicinal plants in the southern slope of Mount Merapi, Yogyakarta, Indonesia. Biodiversitas 20 (8): 2279-2287.

Nahdi MS, Kurniawan AP. 2019b. Ethnobotanical study of medicinal plants in karst environment in Gunu ng Kidul, Yogyakarta, Indonesia. Nusantara Biosci 11(2): 133-141.

Nas J, Nurlinah, Haryanto. 2019. Indigenous village governance: lessons from Indonesia. Publ Administration 2: 94-104.

Ong HG, Ling SM, Win TTH, Kang DH, Lee JH. 2018. Ethnomedicinal plants and traditional knowledge among three Chin indigenous groups in Natma Taung National Park (Myanmar). J Ethnopharmacol 225: 136-158.

Patrick P, Tunnicliffe SD. 2011. What plants and animals do early childhood and primary students' name? Where do they see them? J Sci Educ Technol 20: 630-642.

Prabhu S, Vijayakumar. 2016. Ethnobotanical study of traditionally used medicinal plants in Malayali ethnic people of Pachamalai hills, Tamil Nadu, India. J Pharm Med Res 2 (1): 39-42.

Pradhan D, Suri KA, Pradhan DK, Biswasroy P. 2013. Golden Heart of the nature: Piper betle L. J Pharmacog Phytochem 1 (6): 147-167.

Philips OL. 1996. Some Quantitative Methods for Analyzing Ethnobotanical Knowledge. In: Alexiades MN. 1996. Ethnobotanical Research: A Field Manual. Scientific Publication Departemen the New York Botanical Garden, Bronx, New York.

Putri LSE, Dasumiati, Kristiyanto, Mardiansyah, Malik C, Leuvinadrie LP, Mulyono EA. 2016. Ethnobotanical study of herbal medicine in Ranggawulung Urban Forest, Subang District, West Java, Indonesia. Biodiversitas 17 (1): 172-176.

Ramirez CS. 2007. Ethnobotany and the loss of traditional knowledge in the 21 st century. Ethnobot Res Appl 5: 245-247.

Saynes- Vásquez A, Vibrans H, Vergara-Silva F, Caballero J. 2016. Intracultural differences in local botanical knowledge and knowledge loss among the Mexican Isthmus Zapotecs. PloS One 11 (3): e0151693. DOI: 10.1371/journal.pone.0151693

Silalahi M, Nisyawati, Pandiangan D. 2019. Medicinal plants used by the Batak Toba Tribe in Peadundung Village, North Sumatra, Indonesia. Biodiversitas 20 (2): 510-525.

Song MJ, Kim H, Lee BY, Brian H, Park CH, Hyun CW. 2014. Analysis of traditional knowledge of medicinal plants from residents in Gayasan National Park (Korea). J Ethnobiol Ethnomed 10: 1-47.

Sousa RS, Hanazaki N, Lopes JB, de Barros RFM. 2012. Are gender and age important in understanding the distribution of local botanical knowledge in fishing communities of the Parnaíba delta environmental protection area?. Ethnobot Res Appl 10: 551-559.

Sujarwo W, Arinasa IBK, Salomone F, Caneva G, Fattorini S. 2014. Cultural erosion of Balinese indigenous knowledge of food and nutraceutical plants. Econ Bot 68: 426-437.

Supiandi MI, Mahanal S, Zubaidah S, Julung H, Ege B. 2019. Ethnobotany of traditional medicinal plants used by Dayak Desa Community in Sintang, West Kalimantan, Indonesia. Biodiversitas 20 (5): $1264-1270$

Srithi K, Balslev H, Wangpakapattanawong P, Srisanga P, Trisonthi C. 2009. Medicinal plant knowledge and its erosion among the Mien (Yao) in northern Thailand. Journal of Ethnopharmacology 123: 335342

Suwardi AB, Navia ZI, Harmawan T, Syamsuardi, Mukhtar E. 2020. Ethnobotany and conservation of indigenous edible fruit plants in South Aceh, Indonesia. Biodiversitas 21 (5): 1850-1860.

Syamsurijal. 2014. Islam patuntung: temu-tengkar islam dan tradisi lokal di Tanah Toa Kajang. Urnal. Al-Qalam 20 (2): 171-178. [Indonesian]

Syarif E, Hasriyanti H, Fatchan A, Astina IK, Sumarmi S. 2016a. Conservation values of local wisdom traditional ceremony Rambu Solo Toraja's tribe South Sulawesi as efforts the establishment of character education. EFL J 1 (1): 17-23.

Syarif E, Fatchan A, Sumarmi, Astina IK. 2016b. Tradition of "Pasang RiKajang" in the forests managing in system mores of "Ammatoa" at District of Bulukumba South Sulawesi, Indonesia. Mediter J Soc Sci 7 (6): 325-332.

Tabuti JRS, Dhillion SS, Lye KA. 2003. Traditional medicine in Bulamogi country, Uganda: its practitioners, users, and viability. J Ethnopharmacol 85: 119-129.

Tamalene MN, Al Muhdhar, MHI, Suarsini E, Rohman F. 2016. Medicinal plants study of ethnobotany of medicinal plant of Tobelo 
Dalam (Togutil) ethnic group of Halmahera Island, Indonesia. Med Plants 8: 127-136.

Thomas E, Semo L, Morales M, Noza Z, Nuñez H, Cayuba A, Noza M, Humaday N, Vaya J, Damme PV. 2011. Ethnomedicinal practices and medicinal plant knowledge of the Yuracarés and Trinitarios from indigenous territory and National Park Isiboro-Sécure, Bolivian Amazon. J Ethnopharmacol 133: 153-163.

Trotter RT, Logan MH. 1986. Informant consensus: a new approach for identifying potentially effective medicinal plants. In: Etkin NL (ed) Plants in Indigenous Medicine and Diet: Biobehavioral Approaches. Redgrave Publishing Company, New York.

Tounekti T, Mahdhi M, Khemira H. 2019. Ethnobotanical study of indigenous medicinal plants of Jazan Region, Saudi Arabia. Hindawi Evidence-Based Complement Altern Med https://doi.org/10.1155/2019/3190670

Vandebroek I, Balick MJ. 2012. Globalization and loss of plant knowledge: challenging the paradigm. Plos One 7 (5): e3764.
Wangchuk P, Tobgay T. 2015. Contributions of medicinal plants to the gross national happiness and biodiscovery in Bhutan. J Ethnobiol Ethnomed 11 (48): 1-2.

Weckmüller H, Barriocanal C, Maneja R, Boada M. 2019. Factors a_ecting traditional medicinal plant knowledge of the Waorani, Ecuador. Sustainability 11: 4460.

Wiryono, Wanandi Y, Ilahi AK, Deselina, Senoaji G. 2019. The local knowledge of the plant names and uses by Semende tribe people in Kaur District, Bengkulu Province, Indonesia. Biodiversitas 20 (3): 754-761.

Yakub A, Leksoni AS, Batoro J. 2019. Ethnobotany of medicinal and edible plants of Tobelo Dalam Tribe in Aketajawe Lolobata National Park Area. Indones J Environ Sustain Dev 10 (1): 45-50.

Yirga G, Zeraburk S. 2011. Ethnobotanical study of traditional medicinal plants in Gindeberet District, Western Ethiopia. Proceedings of the 1st International Technology, Education and Environment Conference (c) African Society for Scientific Research (ASSR). Omoku, 8 September 2011. [Nigeria] 